\title{
The variability and reproducibility of whole genome sequencing technology for detecting resistance to anti-tuberculous drugs
}

Jody Phelan ${ }^{1 \dagger}$, Denise M. O'Sullivan ${ }^{2+}$, Diana Machado ${ }^{3+}$, Jorge Ramos ${ }^{3}$, Alexandra S. Whale ${ }^{2}$, Justin O'Grady ${ }^{4}$, Keertan Dheda ${ }^{5}$, Susana Campino ${ }^{1}$, Ruth McNerney ${ }^{5+}$, Miguel Viveiros ${ }^{3 \dagger}$, Jim F. Huggett ${ }^{2,6+}$ and Taane G. Clark ${ }^{1,7^{*}+}$

\begin{abstract}
Background: The emergence of resistance to anti-tuberculosis drugs is a serious and growing threat to public health. Next-generation sequencing is rapidly gaining traction as a diagnostic tool for investigating drug resistance in Mycobacterium tuberculosis to aid treatment decisions. However, there are few little data regarding the precision of such sequencing for assigning resistance profiles.

Methods: We investigated two sequencing platforms (Illumina MiSeq, lon Torrent PGM ${ }^{\top M}$ ) and two rapid analytic pipelines (TBProfiler, Mykrobe predictor) using a well characterised reference strain (H37Rv) and clinical isolates from patients with tuberculosis resistant to up to 13 drugs. Results were compared to phenotypic drug susceptibility testing. To assess analytical robustness individual DNA samples were subjected to repeated sequencing.

Results: The MiSeq and Ion PGM systems accurately predicted drug-resistance profiles and there was high reproducibility between biological and technical sample replicates. Estimated variant error rates were low (MiSeq 1 per 77 kbp, Ion PGM 1 per 41 kbp) and genomic coverage high (MiSeq 51-fold, Ion PGM 53-fold). MiSeq provided superior coverage in GC-rich regions, which translated into incremental detection of putative genotypic drug-specific resistance, including for resistance to para-aminosalicylic acid and pyrazinamide. The TBProfiler bioinformatics pipeline was concordant with reported phenotypic susceptibility for all drugs tested except pyrazinamide and para-aminosalicylic acid, with an overall concordance of $95.3 \%$. When using the Mykrobe predictor concordance with phenotypic testing was $73.6 \%$.

Conclusions: We have demonstrated high comparative reproducibility of two sequencing platforms, and high predictive ability of the TBProfiler mutation library and analytical pipeline, when profiling resistance to first- and second-line anti-tuberculosis drugs. However, platform-specific variability in coverage of some genome regions may have implications for predicting resistance to specific drugs. These findings may have implications for future clinical practice and thus deserve further scrutiny, set within larger studies and using updated mutation libraries.
\end{abstract}

Keywords: Drug resistance, Tuberculosis, Diagnostics, Drug-susceptibility testing, XDR-TB, Next-generation sequencing

\footnotetext{
* Correspondence: taane.clark@|shtm.ac.uk

${ }^{\dagger}$ Equal contributors

'Department of Pathogen Molecular Biology, Faculty of Infectious and

Tropical Diseases, London School of Hygiene \& Tropical Medicine, Keppel

Street, WC1E 7HT London, UK

${ }^{7}$ Faculty of Epidemiology and Population Health, London School of Hygiene \&

Tropical Medicine, WC1E 7HT London, UK

Full list of author information is available at the end of the article
} 


\section{Background}

Mycobacterium tuberculosis, the bacterium that causes tuberculosis disease (TB), has overtaken HIV as the world's major cause of death from an infectious agent [1]. In recent years, control of the disease has been made more difficult by the emergence of multidrug-resistant tuberculosis (MDR-TB), which is resistant to at least rifampicin and isoniazid, and extensively drug-resistant (XDR-TB), which refers to additional resistance to the fluoroquinolones and second-line injectable drugs (amikacin, kanamycin and capreomycin) used to treat MDR-TB [2]. Programmatically incurable TB with resistance to up to 14 drugs has been reported in several parts of the world, including countries with a high TB burden such as India and South Africa [3, 4]. Phenotypic methods of determining susceptibility to anti-TB drugs take weeks or months, they are additively costly, and require culture and manipulation of large numbers of highly infectious bacilli. Drug resistance in $M$. tuberculosis is almost exclusively due to mutations in the circular genome and so molecular determination of resistance offers a rapid, potentially cost effective, and safer alternative. Commercially available molecular-based tests and line probe assays cover a limited number of drugs but, with the exception of rifampicin, they have relatively low sensitivity for detecting all possible molecular targets for resistance [5]. Due to the multiplicity of drugs used in the treatment of $\mathrm{TB}$, determining the full resistance profile for a patient suspected of having drug-resistant disease requires the examination of many loci.

Next-generation whole genome sequencing offers an attractive option as it simultaneously examines all loci and provides information regarding both small and large changes in the genome [5]. This option has been widely reported as a means of identifying putative resistancecausing mutations and more recently has been used in the management of patients with drug-resistant TB to guide selection of appropriate drug regimens [6-11]. This approach is significant because the current treatment outcomes for MDR-TB are poor, largely due to current molecular tests being unable to guide effective individualised therapy. It also has public health implications because of prolonged patient infectiousness due to suboptimal treatment.

The $M$. tuberculosis genome is challenging to sequence due to its high GC content and repetitive nature. Surprisingly, despite the serious consequences of misdiagnosis, there is a paucity of data regarding the reliability of next-generation sequencing platforms or the analytical methodology used for assigning resistance [5]. To address this issue we investigated the utility of two commercial sequencing platforms for predicting resistance to 13 anti-TB drugs. We also examined analytical algorithms and two rapid bioinformatics tools (TBProfiler,
Mykrobe predictor) for predicting resistance from raw sequence data. Testing was performed with a fully susceptible reference strain $(\mathrm{H} 37 \mathrm{Rv})$ and ten clinical isolates from patients with drug-resistant TB.

\section{Methods \\ Samples}

M. tuberculosis clinical isolates were sourced from ten patients with known drug-resistant TB admitted to four different hospitals in Lisbon between 2007 and 2013. These samples were not part of a transmission chain and there is no epidemiological link between the patients. All clinical samples and the reference strain H37Rv (ATCC 25618D-9, Lot \# 60986340) were prepared by inoculating a single colony into Middlebrook 7H9 broth supplemented with 10\% OADC (Becton Dickinson) (see Table 1 for list). Susceptibility testing for the first-line anti-TB drugs rifampicin (RIF), isoniazid (INH), ethambutol (ETB), pyrazinamide (PZA) and streptomycin (STR) and the second-line drugs rifabutin (RFB), amikacin (AMK), capreomycin (CAP), ofloxacin (OFX), moxifloxacin (MOX), ethionamide (ETH), paraaminosalicylic acid (PAS) and linezolid (LZ) was performed on all strains with the MGIT960 system (Becton Dickinson), according to the manufacturer's instructions. Quantitative drug susceptibility testing (qDST) for both first- and second-line drugs was conducted using a combination of the MGIT960 system and the Epicenter V5.80A software equipped with the TB eXIST module (Becton Dickinson) [12, 13].

DNA was extracted and purified from the liquid cultures using a cetyltrimethylammonium bromide (CTAB) method [14]. The quality was assessed by fluorometric quantification, Qubit ${ }^{\mathrm{TM}}$ 3.0 Fluorometer with a dsDNA Broad Range Assay Kit (Thermo Fisher Scientific) and agarose gel electrophoresis. Triplicate DNA samples from each clinical isolate were prepared (biological replicates) and individual DNA extracts were subjected to repeated sequencing (technical replicates).

\section{Library preparation and sequencing}

For MiSeq sequencing, 200 ng of genomic DNA was sheared to an average size of 500 bp by ultrasonication (Covaris S220). Sheared DNA was purified/concentrated on MinElute Spin Columns (Qiagen). DNA concentrations were measured on a Nanodrop UV spectrophotometer and the sheared samples diluted to 5-12.5 $\mathrm{ng} / \mu \mathrm{l}$. Library constructions were performed using the Ovation Rapid DR Multiplex System (NuGen) according to the manufacturer's instructions. Purified libraries were amplified in emulsion PCR, size selected (500$700 \mathrm{bp})$ by preparative electrophoresis on composite gels (1.2\% LMP-Agarose/0.8\% Synergel) and then purified on MinElute Columns. Libraries were sequenced 
Table 1 Study samples (DNA extracted from culture isolates) and their susceptibility to anti-tuberculosis drugs

\begin{tabular}{|c|c|c|c|c|c|c|c|c|c|c|c|c|c|c|c|c|c|c|}
\hline \multirow[t]{2}{*}{ Sample } & \multirow[t]{2}{*}{ Year $^{\mathrm{a}}$} & \multirow[t]{2}{*}{ Lineage } & \multirow{2}{*}{$\begin{array}{l}\text { Spoligo. } \\
\text { family }\end{array}$} & \multicolumn{15}{|c|}{ Drug susceptibility test phenotype } \\
\hline & & & & $\mathrm{INH}$ & RIF & STR & ETB & PZA & RFB & ETH & AMK & CAP & OFX & MOX & PAS & $\mathrm{LZ}$ & KAN $^{\mathrm{b}}$ & Resistance phenotype \\
\hline POR1 & 2007 & 4.3.4.2 & LAM4 & $R$ & $R$ & $\mathbf{R}$ & $R$ & $\mathrm{R}$ & $\mathrm{R}$ & $R$ & $\mathrm{R}$ & $\mathrm{R}$ & $R$ & R & $\underline{R}$ & S & $R$ & XDR-TB \\
\hline POR2 & 2007 & 4.1.1.1 & $x 2$ & $\mathbf{R}$ & $\mathrm{R}$ & S & S & S & R & $\mathrm{R}$ & S & S & S & S & S & S & - & MDR-TB \\
\hline POR3 & 2007 & 4.3.4.2 & LAM1 & $\mathrm{R}$ & $\mathrm{R}$ & $\mathrm{R}$ & $\mathbf{R}$ & $\underline{R}$ & $\mathrm{R}$ & $\mathbf{R}$ & $\mathbf{R}$ & $\mathbf{R}$ & $\mathrm{R}$ & $\mathrm{R}$ & S & S & $\mathbf{R}$ & XDR-TB \\
\hline POR4 & 2007 & 4.3.4.2 & LAM1 & R & $\mathrm{R}$ & R & R & $R$ & R & $\mathrm{R}$ & $\mathbf{R}$ & S & $\mathrm{R}$ & R & S & S & $\mathbf{R}$ & XDR-TB \\
\hline POR5 & 2007 & 4.3.4.2 & LAM4 & R & $\mathrm{R}$ & $\mathbf{R}$ & R & $R$ & R & $\mathrm{R}$ & S & S & S & S & S & S & - & MDR-TB \\
\hline POR6 & 2008 & 4.3.4.2 & LAM4 & $R$ & $\mathrm{R}$ & $\mathbf{R}$ & $\mathrm{R}$ & $\mathrm{R}$ & $R$ & $\mathrm{R}$ & R & $R$ & R & R & S & S & $R$ & XDR-TB \\
\hline POR7 & 2009 & 4.3.4.2 & LAM4 & R & $\mathrm{R}$ & R & R & $R$ & $R$ & $\mathrm{R}$ & R & R & $R$ & R & S & S & $\mathbf{R}$ & XDR-TB \\
\hline POR8 & 2012 & 4.3.4.2 & LAM4 & R & $\mathrm{R}$ & $\mathbf{R}$ & $\mathrm{R}$ & $\mathrm{R}$ & R & $\mathrm{R}$ & R & $\mathrm{R}$ & $\mathrm{R}$ & R & S & S & R & XDR-TB \\
\hline POR9 & 2011 & 4.3.4.2 & LAM4 & $R$ & $R$ & $R$ & $\mathbf{R}$ & $\mathrm{R}$ & $R$ & $R$ & $R$ & $\mathbf{R}$ & R & $R$ & $\underline{R}$ & S & $\mathbf{R}$ & XDR-TB \\
\hline POR10 & 2013 & 4.2 .1 & Ural H3/4 & $\mathrm{R}$ & $\mathrm{R}$ & R & R & $\underline{R}$ & R & $\mathrm{R}$ & S & S & $S$ & $S$ & S & S & $\mathbf{R}$ & MDR-TB \\
\hline H37Rv & - & 4.9 & H37RV & S & S & S & S & S & $S$ & S & $\mathrm{S}$ & S & S & S & $\mathrm{S}$ & $\mathrm{S}$ & - & Pan-susceptible \\
\hline
\end{tabular}

MDR-TB multidrug-resistant TB, XDR-TB extensively drug-resistant TB, INH isoniazid, RIF rifampicin, STR streptomycin, ETB ethambutol, $P Z A$ pyrazinamide, RFB rifabutin, ETH ethionamide, AMK amikacin, CAP capreomycin, OFX ofloxacin, MOX moxifloxacin, PAS para-aminosalicylic acid, LZ linezolid, KAN kanamycin, $S$ "susceptible", $R$ "resistant"

Bold indicates discrepant calls by Mykrobe Predictor, underlining indicates discrepant calls by TBProfiler

aear of collection

${ }^{b}$ Drug susceptibility test not performed, with status inferred by the TBProfiler library

with an Illumina MiSeq V3 and 300-bp paired-end reads with samples randomised across two runs (each $\sim 24 \mathrm{~h}$ in duration).

Ion Torrent library preparation and sequencing was performed at Thermo Fisher Scientific, UK. Sequencing was carried out with the Ion Torrent PGM ${ }^{\mathrm{Tw}}$ system (Ion PGM). Libraries were constructed with the Ion Xpress ${ }^{\text {Th }}$ Plus Fragment Library Kit as per the manufacturer's instructions (MAN0009847 Revision C.0), using $100 \mathrm{ng}$ of genomic DNA which was sheared with the provided Ion Shear $^{\text {Tn }}$ Plus Reagents to an average size of $350 \mathrm{bp}$, size selection using an E-Gel ${ }^{\circ}$ SizeSelect ${ }^{\text {tw }}$ 2\% Agarose Gel, and purification with Agencourt ${ }^{\circ}$ AMPure $^{\circ}$ XP Reagent. Finally, the libraries were quantified on the StepOnePlus ${ }^{\mathrm{Tm}}$ System using the Ion Library Quantitation Kit, then diluted to $100 \mathrm{pM}$ and pooled in equal volume. Purified libraries were sequenced with an Ion $318^{\mathrm{mm}} \mathrm{v} 2$ chip (400-bp kit) and the Ion $\mathrm{PGM}^{\mathrm{Tw}} \mathrm{HiQ}^{\mathrm{m}}$ Chef Kit as stated in the manual (MAN0010919, revision A.0). The runtime was $\sim 3 \mathrm{~h}$ per sample. The software used on both Ion $\mathrm{PGM}^{\mathrm{mi}}$ and the Ion Chef ${ }^{\mathrm{mi}}$ System was Torrent Suite Software version 4.6 .

\section{Bioinformatic pipeline}

For the bioinformatic analysis we used a previously reported pipeline $[10,15,16]$. Unless stated otherwise, software was run at default settings. Reads were trimmed by Trimmomatic using a PHRED quality of 20 as the cutoff. Trimmed reads were then mapped against $\mathrm{H} 37 \mathrm{Rv}$ (GCA_000195955.2) with BWA-mem v0.7.12 [17]. SNP and insertion and deletion (indel) variants were called with Samtools 0.1.19 [18] and GATK v3.6 [19]. We compared the variants called by both algorithms, but also report results of the conservative and typical approach of retaining the consensus polymorphisms across both methods. The genotypes of SNPs were called when an alternative allele was found in $20 \%$ of the mapped reads at a particular position. A default minimum depth of ten reads was required to call SNP genotypes, otherwise genotypes were denoted as missing data. This cutoff has been applied widely $[15,16,20]$. The robustness of the genotype calls was assessed across a range of depths of coverage of the reference and alternative alleles (depth $5-20$, major allelic frequency $>0.5$ and $>0.7$ ). The reference genome was partitioned into overlapping 300-bp sequences allowing the uniqueness of genomic regions to be determined using gem-mappability [21]. Only 1.5\% of the genome was estimated to be non-unique, and variants within these regions were discarded, leaving a set of high quality SNPs and indels. All 36 candidate drug-resistance genes [5] were found to be unique, thus removing the risk of false calling of SNPs due to inappropriate mapping to an analogous region. A summary of the pipeline is presented in Additional file 1: Figure S1.

In silico profiling of $M$. tuberculosis resistance phenotypes We compared two informatics tools for assigning resistance from sequence data. Drug-resistance status across 14 drugs was called in silico from raw sequence data using the web-based TBProfiler tool (http://tbdr.lshtm.ac.uk/) [5]). This tool also generates lists of mutations in candidate loci, and these formed the basis of identifying any additional putative novel polymorphisms. All mutations were checked by analysis of alignments and de novo 
assembly, as well as confirmed by alternative sequencing methods (see the next section, "Confirmation of mutations detected by whole genome sequencing"). Resistance profiles were also generated with the Mykrobe predictor tool (version July 2016) [22].

\section{Confirmation of mutations detected by whole genome sequencing}

Genomic DNA was extracted as described above and used for PCR amplification prior to examination by line probe assay and/or DNA sequencing. The Genotype MTBDRplus (Hain Lifescience) investigates the $r p o B$ and katG genes and inhA regulatory region and Genotype MTBDRsl (version 1, Hain Lifescience) investigates $r r s, \operatorname{gyr} A$ and $e m b B$. Both kits were used according to the manufacturer's instructions. As the line probe assays encompass a limited number of loci, we also performed Sanger sequencing for $i n h A$, katG, tlyA, eis, gidB, pncA, gyrA, ethA, embB, embC-embA, rpsL, folC and thyX genes (see Additional file 2: Table S1 for the primers used). PCR products were purified and both strands sequenced at StabVida (Portugal). All sequences were edited and analysed with ChromasPro 2.0.0 (Technelysium, Australia), compared to the sequences of $M$. tuberculosis H37Rv reference strain (GenBank AL123456.2) and aligned with Clustal Omega [23].

\section{Results}

\section{Coverage}

Triplicate "extraction" DNA samples from ten clinical isolates and a single $\mathrm{H} 37 \mathrm{Rv}$ sample were sequenced on the MiSeq platform. Four DNA samples (from POR5, 6 and 7 and H37Rv) were each sequenced six times ("technical" replicates). Duplicate DNA samples from three clinical isolates (POR1, 2 and 6) were also sequenced on the Ion PGM. Summaries of the sequence data obtained for each platform are presented in Additional file 3: Table S2. With MiSeq sequencing the number of paired reads varied across samples (median 1.2 million, range 0.4 to 3.2 million), and on average $99 \%$ of reads mapped to the H37Rv reference, giving a median depth of coverage of 51-fold (across sample range 18- to 79-fold). The majority of the genome ( $>96 \%)$ was covered to at least tenfold depth.

In contrast, for the Ion PGM platform the median number of reads was 990,854 (range 928,006-1,124,215) translating into a median of 53-fold (range 48- to 59fold) genomic coverage. A large proportion of the genome $(\sim 25 \%)$ had low coverage and was attributed to regions with high GC content (Fig. 1). Whilst high coverage (100- to 200-fold) was attained for regions with GC content up to $69 \%$, above this level coverage drops below tenfold, which was the cutoff used for calling variants. For MiSeq sequence data, this drop only occurs when the GC content reaches $75 \%$ or above. Many regions in the $M$. tuberculosis genome, especially the pe/ ppe genes [24], are high in GC content (median 69\%, range $47-87 \%$ ) and therefore potentially difficult to characterise. The coverage across the 36 drug-resistance candidate genes was high for MiSeq (mean $~ 90$-fold) and exceeded the tenfold cutoff, except in the thy $A$ gene in the three POR1 replicates (Fig. 2). These XDR-TB replicates contained double $d f r A$-thy $A$ deletions, thought to be responsible for para-aminosalicylic acid (PAS) resistance [25]. A direct comparison of the POR1, 2 and 6 sample coverage across platforms highlighted greater variability in candidate genes in Ion PGM due to differential GC content. Whilst there was platform-wide detection of the deletion-driven lower coverage in thy $A$ in POR1 (Fig. 3; Additional file 4: Figure S2), the variable coverage in the neighbouring regions for Ion PGM could lead to less certainty in detection.

\section{SNP variants and error rates}

We estimated the variant error rates (measured as the number of sites which were discordant among replicates) to be low for both platforms (MiSeq 1 per $77 \mathrm{kbp}$, Ion PGM 1 per $41 \mathrm{kbp}$ ). Across comparable samples, the number of high quality SNPs detected using MiSeq data was higher than from Ion PGM, mostly due to low coverage in the alignments generated from the Ion PGM (Additional file 3: Table S2). We sought to investigate the effects of variant calling algorithms on the numbers of SNPs detected in unique genomic regions. From the MiSeq H37Rv data, similar numbers of SNPs were detected across replicates (Samtools 64-69 SNPs and GATK 69-79 SNPs, overlap 69 SNPs), supporting the existence of those variants and high sequence reproducibility (Additional file 5: Table S3). Across clinical isolate replicates the number of SNPs identified was similar and the overlap between variant calling algorithms was high (>90\%; Additional file 5: Table S3). This observation was supported by the Ion PGM data but, due to uneven coverage, at least 120 SNPs fewer were identified when compared to matching MiSeq samples. Within platforms and calling algorithms there was variation between replicates in the indels detected, but there was high overlap between algorithms ( $>90 \%$; Additional file 5: Table S3). Compared to SNPs the breakpoints for these variants are more difficult to characterise from alignments.

For the MiSeq platform data we assessed the number of SNP genotypes called across a range of coverage depths of the reference and alternative alleles (total depth 5- to 20-fold; major allelic frequency $>0.5$ and >0.7). The number of SNPs decreased pseudolinearly with decreasing minimum read depth for $\mathrm{H} 37 \mathrm{Rv}$ (87 to 67 SNPs; Additional file 6: Figure S3) and the ten clinical isolates (2290 to 2097 SNPs; Additional file 7: 


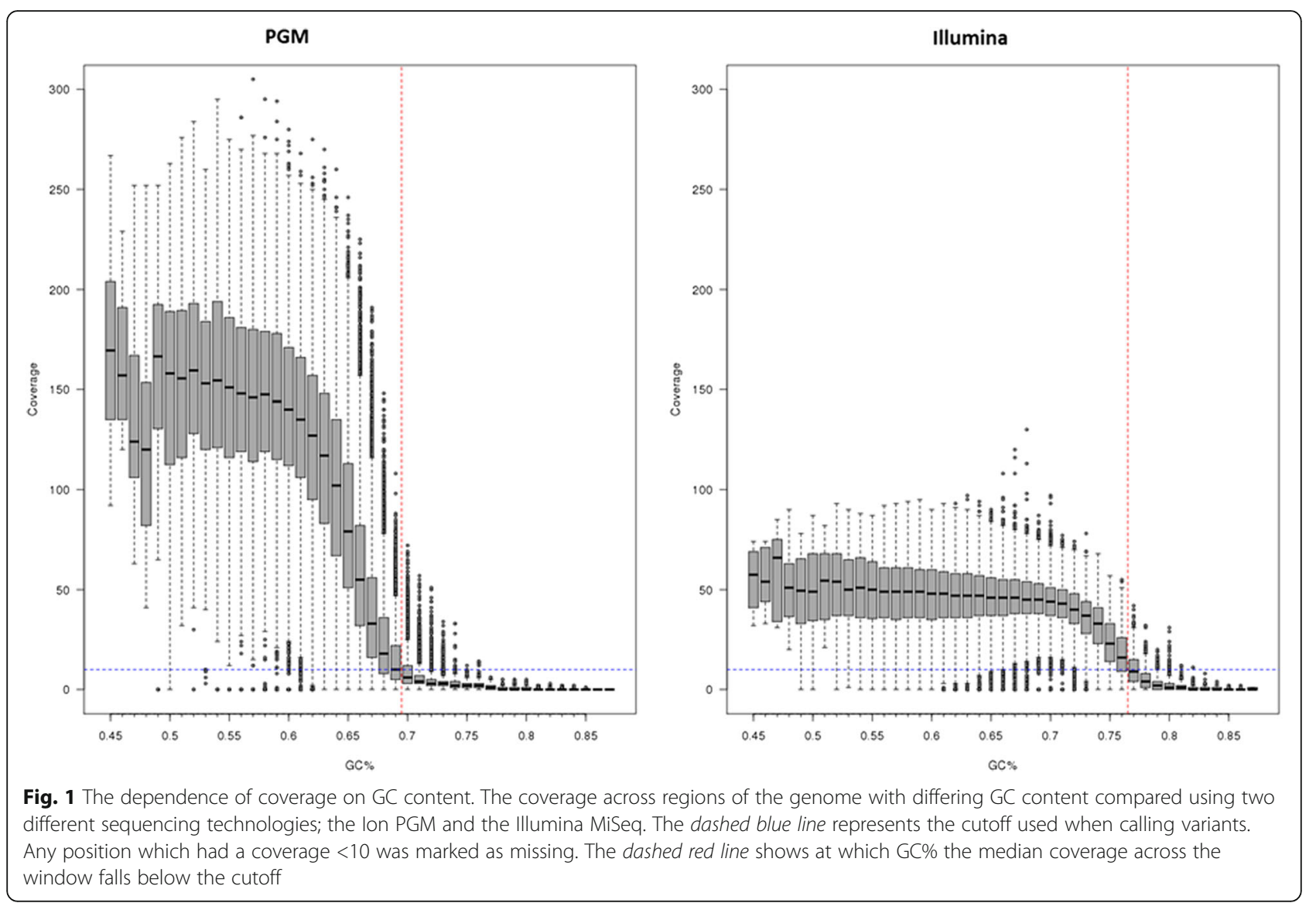

Figure S4). In general, differences in the number of SNPs between the Samtools and GATK algorithms decreased as the depth of coverage and allelic frequency thresholds increased. For H37Rv, read depths in excess of 20 -fold had no impact on variants detected. Across clinical isolates, the highest possible stringency tested consisted of using a minimum coverage of 20 and an allelic frequency of 0.7 and led to near identical numbers of total SNPs called by the two variant calling algorithms (Samtools 1943, GATK 1990, either 2097, both 1840 SNPs; Additional file 7: Figure S4). Much of the discordance in the number of SNPs within replicate groups is due to differences in coverage leading to some polymorphisms not passing quality control filters. Using SNPs for which all replicates have nonmissing genotypes, all replicates had identical numbers of SNPs except POR3C, which differed by two SNPs between POR3A and POR3B. Overall, the analyses indicated no major differences in SNPs detected between the two calling algorithms, and this supported the use of consensus variants for downstream analysis. For example, the set of common SNP variants were used to cluster all samples within a phylogenetic tree using FastTree v2.1.7 [26] (Additional file 8: Figure S5). Perfect clustering was observed amongst isolates and their replicates. At a finer resolution, we analysed the SNP differences between the replicates, and none were identified.

\section{Calling in silico resistance phenotypes}

When the MiSeq raw sequence data were subjected to analysis using TBProfiler, agreement with phenotypic susceptibility testing was high $(95.3 \%, 82 / 86$; Table 1$)$. Discrepant results were recorded for PZA $(\times 2)$ and PAS $(\times 2)$ where phenotypically resistant isolates not identified by TBProfiler were found to have novel mutations in known candidate genes (Additional file 9: Table S4). The novel polymorphisms included a deletion in $p n c A$ of $20 \mathrm{bp}$ (nucleotides 437-449) and a nucleotide insertion (GG) between codons 130 and 131. PAS-resistant isolates had a folC S98G mutation and a thyX G-4A, thyX I161T, dfrA-thyA deletion. Phenotypic testing of kanamycin drug susceptibility was not performed, but mutations associated with its resistance were detected in all eight isolates (Table 1; Additional file 9: Table S4). All mutations were confirmed using independent Sanger capillary sequencing and/or the line probe assays Genotype MTBDRplus and Genotype MTBDRsl (Hain). 


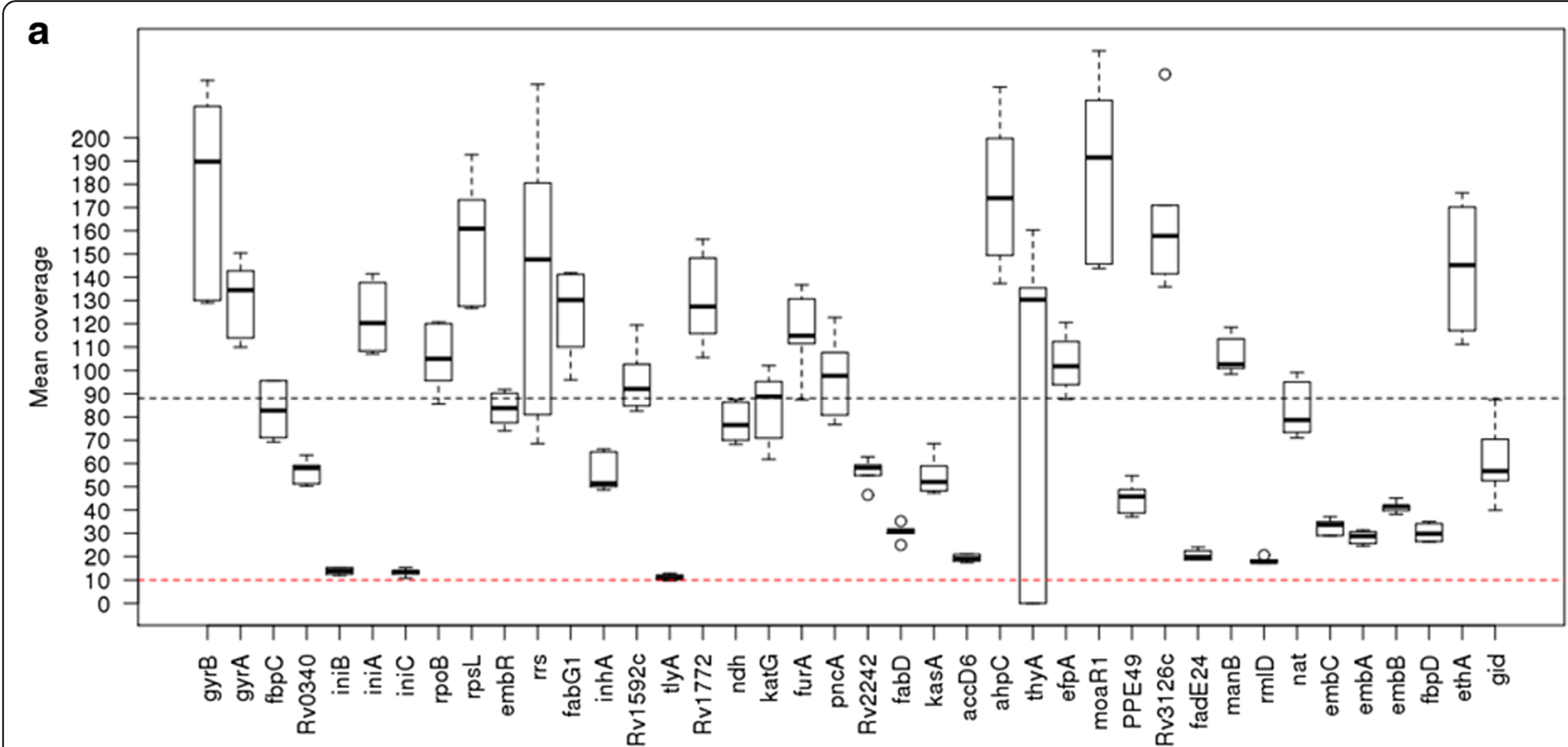

b

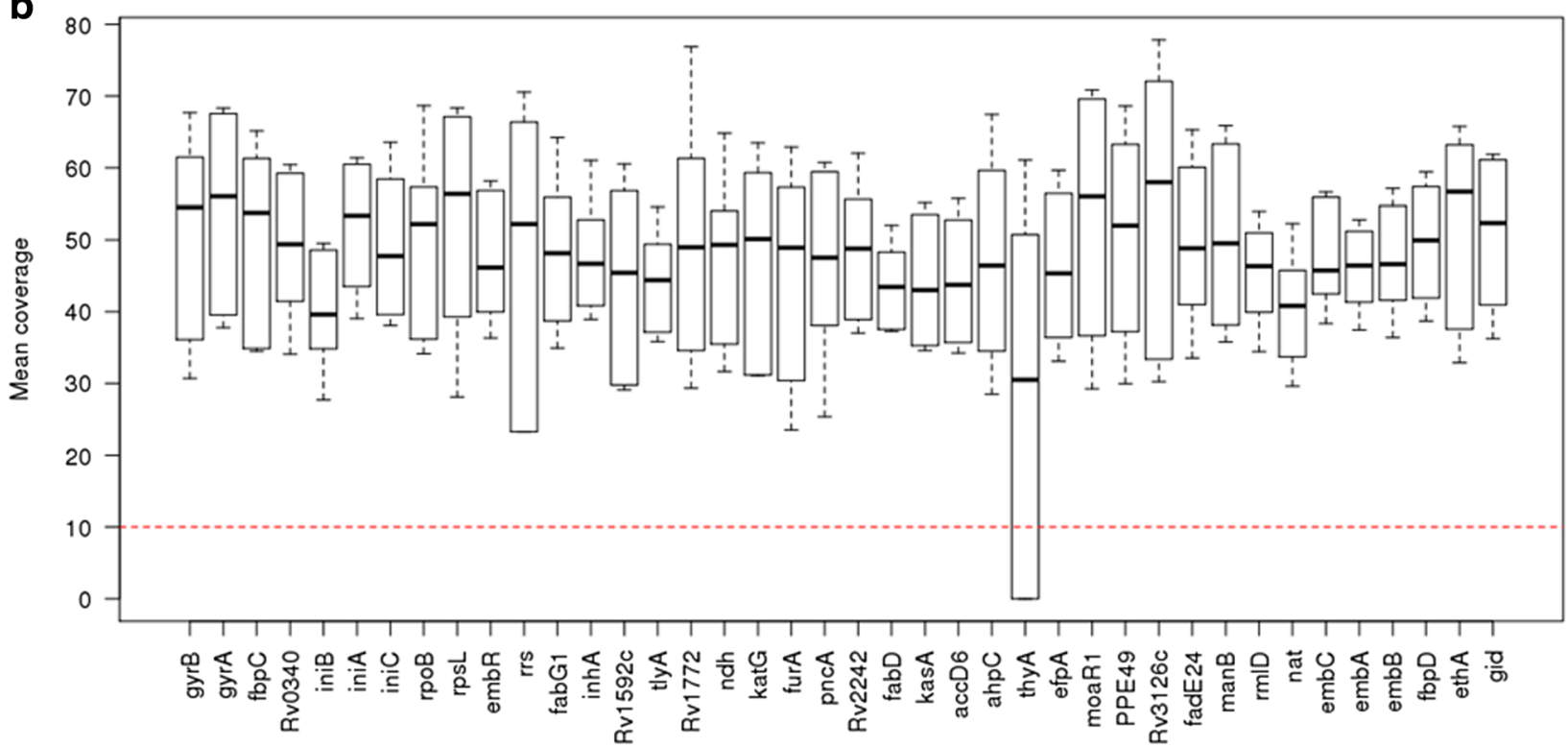

Fig. 2 Coverage across drug-resistance genes. The coverage across the drug-resistance genes in POR1, 2 and 6 samples sequenced using both the $\mathbf{a}$ Ion PGM and $\mathbf{b}$ Illumina MiSeq. The dashed red line represents the cutoff used when calling variants. Any position with less than tenfold coverage was marked as missing. The low coverage in thyA is due to a deletion polymorphism

Phenotypic resistance profiles were confirmed and quantified by the qDST method for the MGIT960 system $[12,13]$.

The Mykrobe predictor tool was also applied to in silico call resistance. This approach looks for mutations associated with resistance to first-line drugs (rifampicin, isoniazid, ethambutol) and second-line drugs (streptomycin, ciprofloxacin, ofloxacin, moxifloxacin, amikacin, kanamycin, capreomycin). Of the 72 resistance calls made, 19 (26.4\%) were incorrectly called "susceptible".
False negative calls were made for isoniazid $(\times 1)$, ethambutol $(\times 2)$, streptomycin $(\times 4)$, amikacin $(\times 4)$, and capreomycin $(\times 3)$. Additionally there was a disagreement between TBprofiler and Mykrobe predictor with four samples for kanamycin, the latter program calling them as "susceptible" (Table 1).

For Ion PGM, when predicting individual drugresistance profiles in the three isolates, in one isolate the gyrA D94A mutation associated with fluoroquinolone resistance could not be detected due to lack of coverage 


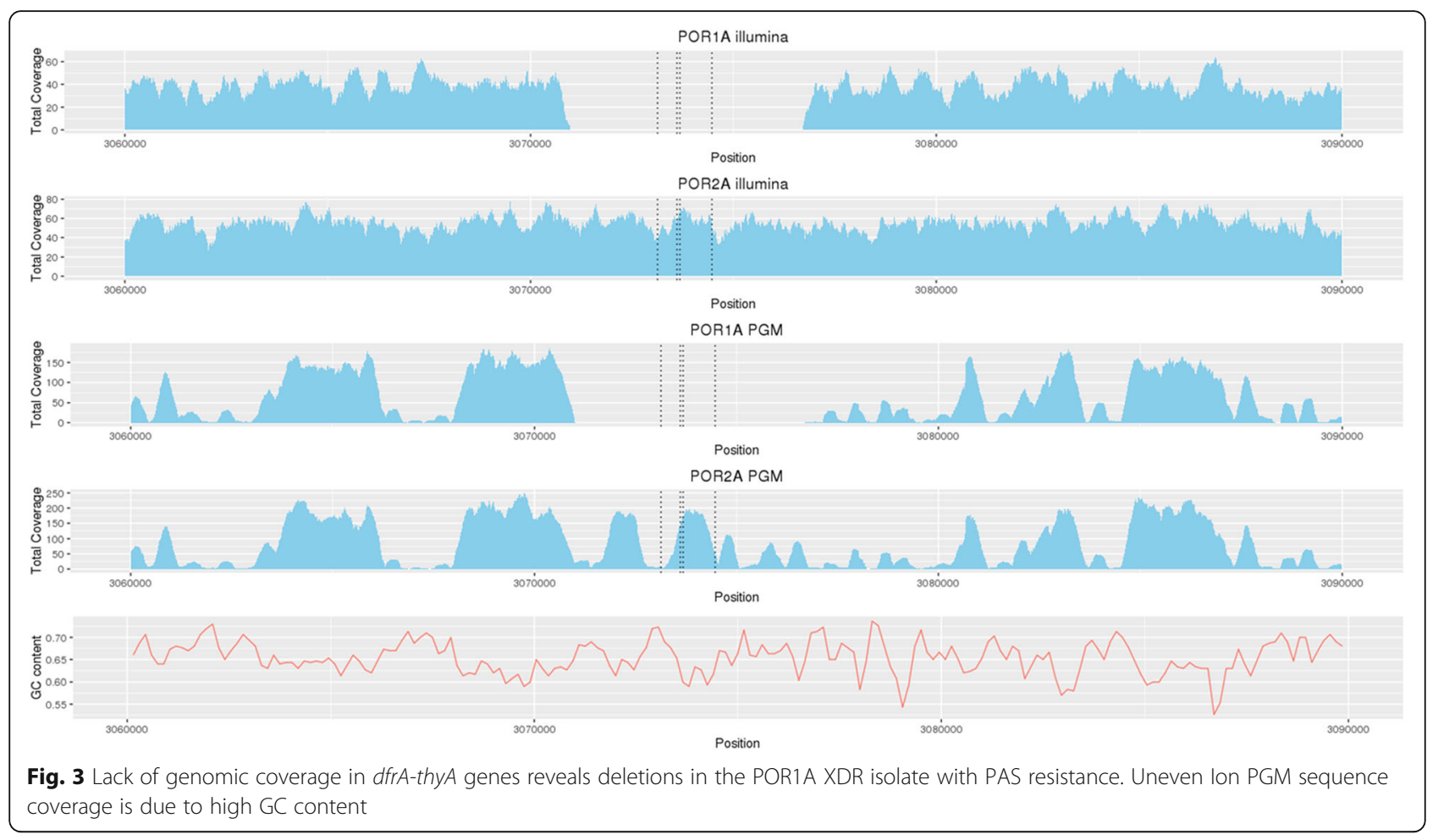

(Additional file 5: Table S3). However, the mutation was recovered if the coverage threshold was relaxed from ten- to fourfold.

\section{Discussion}

Advances in next-generation sequencing technology have expanded opportunities for genome analysis in the clinical laboratory. Determining resistance to anti-TB drugs by whole genome sequencing has been demonstrated as feasible and is being implemented in some specialist centres [6]. For acceptance as a diagnostic tool to guide treatment of drug-resistant TB the sequencing platforms and analytical tools employed must be robust and reliable. Here we have investigated the performance of two commercial 'bench-top' next generation sequencing platforms and attempted to assess the robustness of a bioinformatics analysis pipeline with respect to variant calling, across sequencing replicates.

The MiSeq and Ion PGM both proved satisfactory for determining drug-resistance profiles. Compared to Ion PGM, MiSeq sequence coverage was more uniform and was better represented in regions with high GC content. However, we did not investigate the impact of the different library preparation methods used (mechanical (MiSeq) and enzymatic (Ion PGM) processing). Sample quality and the mode or preparation have been shown to influence the depth of coverage in high GC regions [27], and further work is required to investigate this. The Ion PGM platform has previously been used to characterise mutations in XDR-TB strains [6], but the minimum read depth used to call alleles (fourfold) were less stringent than the tenfold coverage threshold adopted here.

Samtools and GATK when used to process the raw sequence data produced diverse outputs but filtering based on coverage and allelic frequency led to almost complete agreement on resistance causing SNPs. There was, however, lower concordance between the final sets of indels. As previously reported, the false discovery rate for Samtools is higher than for GATK and rises as coverage increases [28]. A common strategy is to undertake dual analysis and consider the intersection of the Samtools and GATK derived SNPs but select only the GATK indels [16]. The high reproducibility of sequence data from replicate samples is reassuring as it affirms the validity of next-generation sequencing as a tool for investigating transmission events.

Of the two rapid tools examined, the TBProfiler gave $100 \%$ concordance with phenotypic DST results for INH, RIF, STR, ETB, ETH and the fluoroquinolones. Of the nine PZA-resistant isolates, known resistance SNPs were reported for seven isolates with an insertion and deletion observed for the remaining two. Possible novel resistance mutations were also observed for both the PAS-resistant isolates. The Mykrobe predictor detected resistance for nine drugs, of which eight had DST results. Concordance was $100 \%$ for RIF, OFX and MOX, but resistance was missed for one or more isolates for 
the remaining five drugs. Misclassification of resistance of amikacin and capreomycin as susceptible has significant clinical implications as patients may be assigned treatment that is not effective for XDR-TB.

The identification of a PAS resistance-related $d f r A$ thyA double deletion in an XDR-TB sample highlights the need to look at non-SNP variants. Significantly, the laboratory platform being used may impact the detection of putative drug resistance. This is critical in XDR-TB and resistance beyond XDR-TB where use of drugs like PAS may make the difference in providing a life-saving effective regimen of at least five drugs [29]. Large deletions and other structural variants may be detected by applying a combination of complementary approaches (pair-end, split-read and depth of coverage) followed by a validation process involving de novo assembly of bordering reads and re-alignment to the reference genome $[10,16,24]$. However, high genome-wide sequence coverage is necessary to perform such analyses.

As expected the genotypic profiling was concordant with the phenotypic determination of drug-resistance levels confirming the reliability and robustness of the selected genes and mutations as predictors of resistance for almost all drugs tested; with discrepancies still being noticed for PZA and PAS due to lack of enough information on their mechanism of action [12, 30]. Surprisingly, no discrepancies were found for EMB, a drug known to have low correlation between the emb genes and phenotypic resistance [12].

\section{Conclusions}

Sequencing platforms are becoming more accessible and economical. Our work suggests that they are capable of delivering high quality data regarding resistance to anti-TB drugs but do not all perform to the same standard and quality monitoring is advisable. Further studies are needed to evaluate these analytical tools, which as yet do not have regulatory approval for clinical use. It is expected that drugresistance profiling using next-generation sequencing will gain accuracy and reliability with the gathering of improved knowledge of the drug-target genes and resistance-causing mutations, including for the new drugs recently approved for the treatment of MDR- and XDR-TB [29, 31]. Ultimately, drug resistance profiling using next-generation sequencing offers rapid assessment of resistance-associated mutations, thus accelerating access to effective treatment.

\section{Additional files}

Additional file 1: Figure S1. Bioinformatics pipeline. (TIFF 81 kb)

Additional file 2: Table S1. Sanger sequencing primers for genomic variant confirmation. (DOCX $132 \mathrm{~kb}$ )

Additional file 3: Table S2. Summary of the sequencing data, coverage and SNPs for each sample. (DOCX $22 \mathrm{~kb}$ )
Additional file 4: Figure S2. a Mean coverage for all samples for each drug resistance gene. Deletion of $d f r A-t h y A$ is evident by the zero coverage outliers in POR1. b Mean coverage across drug-resistance genes. (TIFF $273 \mathrm{~kb}$ )

Additional file 5: Table S3. Replicate variation across extraction and calling algorithms, and phenotypic profiles. (DOCX $20 \mathrm{~kb}$ )

Additional file 6: Figure S3. The changes in the number of SNPS characterised across algorithms for H37Rv. (TIFF $85 \mathrm{~kb}$ )

Additional file 7: Figure S4. The changes in the number of SNPS characterised across algorithms for the ten clinical isolates. (TIFF $92 \mathrm{~kb}$ )

Additional file 8: Figure S5. Phylogenetic tree of all the MiSeq sequenced samples. (TIFF $76 \mathrm{~kb}$ )

Additional file 9: Table S4. Mutations that potentially explain drug resistance in the samples. (DOCX $19 \mathrm{~kb}$ )

\section{Acknowledgements}

We wish to thank ThermoFisher Scientific for performing the lon Torrent $P G M^{T M}$ sequencing. Sequence analysis was performed on the MRC UK funded eMedlab computing resource.

\section{Funding}

This work was supported by the UK National Measurement System and the European Metrology Research Programme (EMRP) joint research project [HLT08] "Infect-Met", which is jointly funded by the EMRP participating countries within EURAMET and the European Union. JP is the recipient of a BBSRC PhD studentship. TGC is funded by the Medical Research Council UK (grant number MR/K000551/1, MR/M01360X/1, MR/N010469/1). SC is funded by the Medical Research Council UK (grant number MR/M01360X/1). DM is supported by Fundação para a Ciência e a Tecnologia, Portugal (grant number SFRH/BPD/100688/2014). DM, JR and MV are thankful for the support of grant GHTMUID/Multi/04413/2013 from Fundação para a Ciência e a Tecnologia, Portugal. These funding bodies did not have a role in the design of the study and collection, analysis and interpretation of data and in writing the manuscript.

Availability of data and materials

All raw sequencing data for both MiSeq and Ion PGM can be accessed from the European Nucleotide Archive (ENA) Sequence Read Archive (project accession PRJEB15555). Pipeline scripts are available from http://pathogenseq.lshtm.ac.uk

\section{Authors' contributions}

JP, DMO, MV, JFH and TGC conceived and designed the study; DMO, ASW, $\mathrm{DM}$ and JR performed laboratory experiments and curation of meta data for sequencing; DMO, DM, JR, ASW, JO'G, SC, MV and JFH contributed biological samples, sequencing or phenotypic data; JP performed the statistical analysis under the guidance of SC and TGC; DMO and JFH led the sequencing efforts; JP, DMO, DM, KD, RM, MV, JFH and TGC wrote/drafted and finalised the manuscript with contributions from all other authors. The final manuscript was read and approved by all authors.

\section{Competing interests}

The authors declare that they have no competing interests.

Consent for publication

Not applicable.

Ethics approval and consent to participate

Not applicable.

\section{Author details}

${ }^{1}$ Department of Pathogen Molecular Biology, Faculty of Infectious and Tropical Diseases, London School of Hygiene \& Tropical Medicine, Keppel Street, WC1E 7HT London, UK. ${ }^{2}$ Molecular Biology, LGC Ltd, Queens Road, Teddington, Middlesex TW11 OLY, UK. ${ }^{3}$ Unidade de Microbiologia Médica, Global Health and Tropical Medicine, GHTM, Instituto de Higiene e Medicina Tropical, IHMT, Universidade NOVA de Lisboa, UNL, Lisbon, Portugal.

${ }^{4}$ Norwich Medical School, University of East Anglia, Norwich Research Park, Norwich NR4 7TJ, UK. 'Division of Pulmonary Medicine and UCT Lung Institute, Lung Infection and Immunity Unit, University of Cape Town, Groote Schuur Hospital, Observatory, 7925, Cape Town, South Africa. ${ }^{6}$ School of 
Biosciences \& Medicine, Faculty of Health \& Medical Science, University of Surrey, Guildford GU2 7XH, UK. ${ }^{7}$ Faculty of Epidemiology and Population Health, London School of Hygiene \& Tropical Medicine, WC1E 7HT London, UK

Received: 30 August 2016 Accepted: 30 November 2016

Published online: 22 December 2016

\section{References}

1. World Health Organization. Global Tuberculosis Report 2015. Geneva: World Health Organization; 2015.

2. Zignol M, Dean AS, Falzon D, van Gemert W, Wright A, van Deun A, et al. Twenty years of global surveillance of antituberculosis-drug resistance. N Engl J Med. 2016;375:1081-9.

3. Dheda K, Gumbo T, Gandhi NR, Murray M, Theron G, Udwadia Z, et al. Global control of tuberculosis: from extensively drug-resistant to untreatable tuberculosis. Lancet Respir Med. 2014;2:321-38.

4. Pietersen E, Peter J, Streicher E, Sirgel F, Rockwood N, Mastrapa B, et al. High frequency of resistance, lack of clinical benefit, and poor outcomes in capreomycin treated South African patients with extensively drug-resistant tuberculosis. PLoS One. 2015;10:e0123655.

5. Coll F, McNerney R, Preston M, Guerra-Assunção JA, Warry A, Hill-Cawthorn $G$, et al. Rapid determination of anti-tuberculosis drug resistance from whole-genome sequences. Genome Med. 2015;5:51.

6. Witney AA, Gould KA, Arnold A, Coleman D, Delgado R, Dhillon J, et al. Clinical application of whole-genome sequencing to inform treatment for multidrug-resistant tuberculosis cases. J Clin Microbiol. 2015;53:1473-83.

7. Zhang H, Li D, Zhao L, Fleming J, Lin N, Wang T, et al. Genome sequencing of 161 Mycobacterium tuberculosis isolates from China identifies genes and intergenic regions associated with drug resistance. Nat Genet. 2013;45:1255-60.

8. Clark TG, Mallard K, Coll F, Preston M, Assefa S, Harris D, et al. Elucidating emergence and transmission of multidrug-resistant tuberculosis in treatment experienced patients by whole genome sequencing. PLoS One. 2013;8:e83012

9. Farhat MR, Sultana R, lartchouk O, Bozeman S, Galagan J, Sisk P, et al. Genetic determinants of drug resistance in mycobacterium tuberculosis and their diagnostic value. Am J Respir Crit Care Med. 2016. doi:10.1164/ rccm.201510-20910C.

10. Phelan J, Coll F, McNerney R, Ascher DB, DE Pires V, Furnham N, et al. Mycobacterium tuberculosis whole genome sequencing and protein structure modelling provides insights into anti-tuberculosis drug resistance. BMC Med. 2016;14:31.

11. Brown AC, Bryant JM, Einer-Jensen K, Holdstock J, Houniet DT, Chan JZM, et al. Rapid Whole Genome Sequencing of M. tuberculosis directly from clinical samples. J Clin Microbiol. 2015. doi:10.1128/JCM.00486-15.

12. Cambau E, Viveiros M, Machado D, Raskine L, Ritter C, Tortoli E, et al. Revisiting susceptibility testing in MDR-TB by a standardized quantitative phenotypic assessment in a European multicentre study. J Antimicrob Chemother. 2015;70:686-96.

13. Springer B, Lucke K, Calligaris-Maibach R, Ritter C, Bottger EC. Quantitative drug susceptibility testing of Mycobacterium tuberculosis by use of MGIT 960 and EpiCenter instrumentation. J Clin Microbiol. 2009;47:1773-80.

14. Larsen MH, Biermann K, Tandberg S, Hsu T, Jacobs WR, Larsen MH. Genetic manipulation of Mycobacterium tuberculosis. Curr Protoc Microbiol. 2007;8:10A.2.1

15. Benavente ED, Coll F, Furnham N, McNerney R, Glynn JR, Campino S, et al. PhyTB: Phylogenetic tree visualisation and sample positioning for $\mathrm{M}$ tuberculosis. BMC Bioinformatics. 2015;16:155.

16. Coll F, Preston M, Guerra-Assunção JA, Hill-Cawthorn G, Harris D, Perdigão J, et al. PolyTB: a genomic variation map for Mycobacterium tuberculosis. Tuberculosis (Edinb). 2014;94:346-54.

17. Li H. Toward better understanding of artifacts in variant calling from high-coverage samples. Bioinformatics. 2014;30:2843-51.

18. Li H. A statistical framework for SNP calling, mutation discovery, association mapping and population genetical parameter estimation from sequencing data. Bioinformatics. 2011:27:2987-93.

19. McKenna A, Hanna M, Banks E, Sivachenko A, Cibulskis K, Kernytsky A, et al. The Genome Analysis Toolkit: A MapReduce framework for analyzing next-generation DNA sequencing data. Genome Res. 2010;20:1297-303.
20. Preston MD, Campino S, Assefa SA, Echeverry DF, Ocholla H, Amambua-Ngwa A, et al. A barcode of organellar genome polymorphisms identifies the geographic origin of Plasmodium falciparum strains. Nat Commun. 2014. doi:10.1038/ ncomms5052.

21. Derrien T, Estellé J, Marco Sola S, Knowles DG, Raineri E, Guigó R, et al. Fast computation and applications of genome mappability. PLoS One. 2012;7:e30377.

22. Bradley P, Gordon NC, Walker TM, Dunn L, Heys S, Huang B, et al. Rapid antibiotic-resistance predictions from genome sequence data for Staphylococcus aureus and Mycobacterium tuberculosis. Nat Commun. 2015;6:10063.

23. Sievers F, Wilm A, Dineen D, Gibson TJ, Karplus K, Li W, et al. Fast, scalable generation of high-quality protein multiple sequence alignments using Clustal Omega. Mol Syst Biol. 2011;7:539.

24. Phelan JE, Coll F, Bergval I, Anthony RM, Warren R, Sampson SL, et al. Recombination in pe/ppe genes contributes to genetic variation in Mycobacterium tuberculosis lineages. BMC Genomics. 2015;17:151.

25. Moradigaravand D, Grandjean L, Martinez E, Li H, Zheng J, Coronel J, et al. dfrA thyA Double deletion in para-aminosalicylic acid-resistant Mycobacterium tuberculosis Beijing strains. Antimicrob Agents Chemother. 2016;60:3864-7.

26. Price MN, Dehal PS, Arkin AP. FastTree: computing large minimum evolution trees with profiles instead of a distance matrix. Mol Biol Evol. 2009:26:1641-50.

27. Tyler AD, Christianson S, Knox NC, Mabon P, Wolfe J, Van Domselaar G, et al. Comparison of sample preparation methods used for the next-generation sequencing of Mycobacterium tuberculosis. PLoS One. 2016;11:e0148676.

28. Liu X, Han S, Wang Z, Gelernter J, Yang B-Z, Nielsen R, et al. Variant callers for next-generation sequencing data: a comparison study. PLoS One. 2013:8:e75619.

29. WHO. WHO treatment guidelines for drug-resistant tuberculosis. 2016. http://www.who.int/tb/areas-of-work/drug-resistant-tb/treatment/resources/en/. Accessed 10 Oct 2016

30. Domínguez J, Boettger EC, Cirillo D, Cobelens F, Eisenach KD, Gagneux S, et al. Clinical implications of molecular drug resistance testing for Mycobacterium tuberculosis: a TBNET/RESIST-TB consensus statement. Int J Tuberc Lung Dis. 2016:20:24-42.

31. Papaventsis D, Casali N, Kontsevaya I, Drobniewski F, Cirillo DM, Nikolayevskyy V. Whole genome sequencing of $\mathrm{M}$. tuberculosis for detection of drug resistance: a systematic review. Clin Microbiol Infect. 2016. doi:10.1016/j.cmi.2016.09.008.

\section{Submit your next manuscript to BioMed Central and we will help you at every step:}

- We accept pre-submission inquiries

- Our selector tool helps you to find the most relevant journal

- We provide round the clock customer support

- Convenient online submission

- Thorough peer review

- Inclusion in PubMed and all major indexing services

- Maximum visibility for your research

Submit your manuscript at www.biomedcentral.com/submit
) Biomed Central 